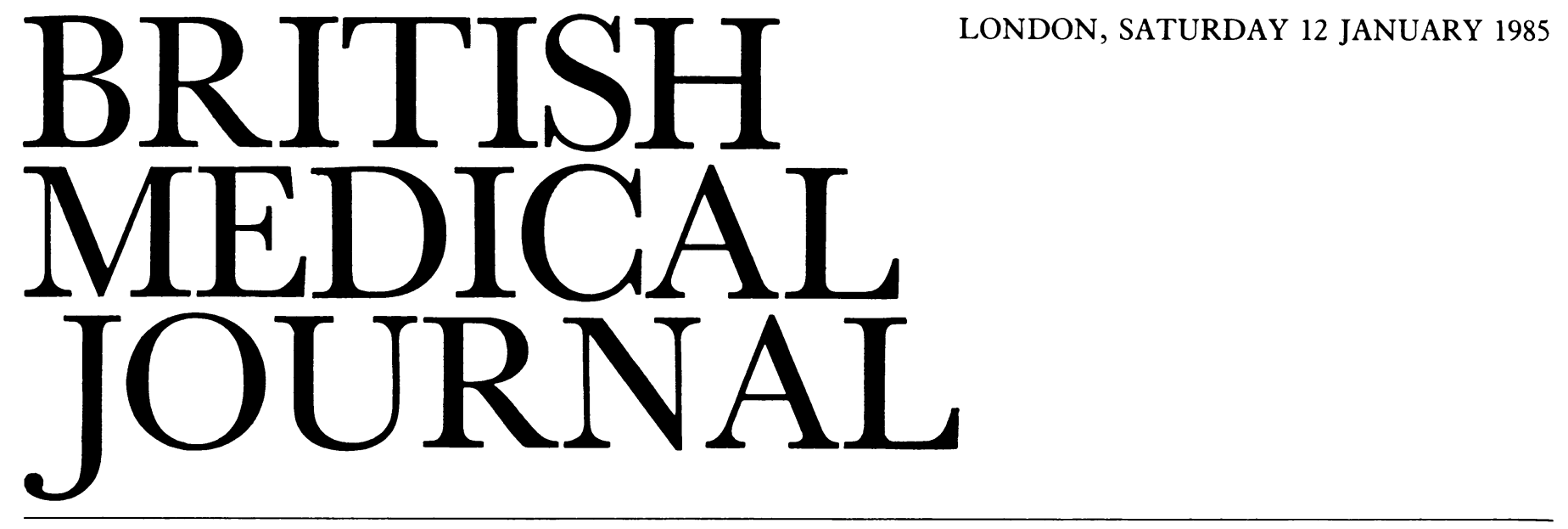

\title{
Sudden cardiac death and acute coronary thrombosis
}

Most deaths caused by coronary artery disease occur suddenly outside hospital, beyond the reach of the coronary care unit. ${ }^{12}$ Experience in resuscitation schemes outside hospital has identified ventricular fibrillation as the most common arrhythmia in "sudden death," though asystole or profound bradycardia may also occur. ${ }^{3}$ As is the case when ventricular fibrillation occurs within the coronary care unit, successful resuscitation from fibrillation outside hospital commonly leads to long term survival. ${ }^{4}$ Investigation of this group of survivors of "sudden death" has shown a high incidence of severe multivessel coronary artery disease ${ }^{5}$ and readily inducible ventricular tachycardia or fibrillation, ${ }^{6}$ though only a third of the patients had evidence of established acute myocardial infarction. ${ }^{+}$

Might sudden cardiac death, then, be a different disease from acute myocardial infarction? Recent angiographic studies in acute myocardial infarction have shown occlusion of the coronary arteries in almost all patients. ' In contrast, some pathological studies of sudden cardiac death have found that coronary thrombi are present in fewer than $20 \%$ of cases. ${ }^{89}$ This view has recently been challenged by Davies and Thomas in a painstaking necropsy study of 100 victims who died within six hours of the onset of symptoms. ${ }^{10}$ All had, by definition, one or more coronary artery stenoses of at least $75 \%$ of the luminal area $(50 \%$ diameter). Intraluminal coronary thrombi were present in 74 , with no difference in the incidence of thrombi between those who died within 15 minutes of the onset of symptoms and those who died after one hour or more. Of the 26 without intraluminal thrombosis, a fresh thrombus within the intima was found in 21 , associated with fissuring of an atherosclerotic plaque in 19. Only five had no acute arterial lesion. Davies and Thomas did not distinguish between "instantaneous" death (within 30 seconds) and "sudden" death (occurring within 15 minutes). One study has shown a low incidence of thrombi in instantaneous death, and it remains possible that a primary electrical event rather than arterial occlusion is the mechanism in these cases. ${ }^{11}$

These findings suggest that the pathogenesis of sudden, but not necessarily instantaneous, cardiac death is an acutely evolving arterial obstruction superimposed on a chronic, stenotic atherosclerotic plaque. The resulting myocardial ischaemia gives rise to complex biochemical and electrophysiological changes, which in most instances result in ventricular fibrillation. ${ }^{12}$ Nearly half the intraluminal thrombi found by Davies and Thomas occupied less than $50 \%$ of the area of the lumen, and the critical reduction of coronary blood flow must have been the result of another mechanism such as superadded spasm or distal platelet embolisation. In this group acute myocardial infarction might possibly not have developed if resuscitation had been successful. The tendency to spontaneous recanalisation of occluded arteries within a few hours has been shown in acute myocardial infarction, and is likely also to occur in survivors of sudden death.

What are the clinical implications of these studies on sudden cardiac death? At present the only treatment available is cardiopulmonary resuscitation and defibrillation. Excellent results have been reported from those centres in which effective schemes of out of hospital resuscitation have been introduced. ${ }^{34}$ Antiarrhythmic treatment guided by intracardiac electrophysiological studies reduces the recurrence of malignant ventricular arrhythmia in those patients with inducible ventricular tachycardia or fibrillation. ${ }^{6} \mathrm{~A}$ few patients with multiple episodes of refractory ventricular tachycardia or fibrillation may benefit from an automatic implantable cardioverter/defibrillator. ${ }^{13}$ The apparent paradox of the failure of primary prophylaxis with conventional antiarrhythmic drugs in unselected patients after acute myocardial infarction ${ }^{14}$ is attributable to the substantial change that occurs in the properties of these drugs in hypoxic myocardium ${ }^{15}$ - with the production of a proarrhythmic effect in many instances. ${ }^{16}$ The success of the $\beta$ adrenergic blocking agents in secondary prevention trials after myocardial infarction is most probably due to their anti-ischaemic rather than any antiarrhythmic actions. ${ }^{17-19}$

The two logical approaches to the primary prevention of sudden death would be to retard or prevent the development of atherosclerotic coronary disease and to reduce the thrombogenicity of blood in susceptible people. The recent Lipid Research Clinics study did not specifically address the relation between sudden death and lowering plasma cholesterol..$^{20}$ Prevention of coronary thrombosis by anticoagulation with warfarin has been extensively studied with contradictory results, though the Dutch Sixty Plus study provided some indirect positive evidence. ${ }^{21}$ Of the antiplatelet agents, aspirin is the most promising contender. ${ }^{22}$

In summary, the results of Davies and Thomas lead us to 
the common sense conclusion that the prevention of sudden cardiac death and that of acute myocardial infarction are likely to be achieved by the same measures. Until primary prevention can abolish the problem we may save a substantial number of lives by investing more heavily in schemes for out of hospital resuscitation.

STUART M COBBE

Clinical Reader and Honorary Consultant Cardiologist,

John Radcliffe Hospital,

Oxford OX3 9DU

1 Armstrong A, Duncan B, Oliver MF, et al. Natural history of acute coronary heart attacks. A community study. Br Heart $\mathcal{f}$ 1972;34:67-80.

McNeilly RH, Pemberton J. Duration of the last attack in 998 fatal cases of coronary artery disease and its relation to possible cardiac resuscitation. Br Med $\mathcal{J} 1968$;iii:139-42.

3 O'Doherty M, Tayler DI, Quinn E, Vincent R, Chamberlain DA. Five hundred patients with

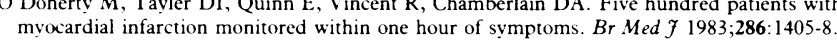
myocardial infarction monitored within one hour of symptoms. Br Med f 1983;286:1405-8.
4 Cobb LA, Baum RS, Alvarez. H, Schaffer WA. Resuscitation from out-of-hospital ventricular Cobb LA, Baum RS, Alvarez. H, Schaffer WA. Resuscitation from out-of-
fibrillation: 4 years' follow-up. Circulation 1975;51,52 (suppl III):223-35.

fibrillation: 4 years' follow-up. Circulation 1975;51,52 suppl III):223-35.
5 Weaver WD, Lorch GS, Alvarez HA, Cobb LA. Angiographic findings and prognostic Weaver WD, Lorch GS, Alvarez HA, Cobb LA. Angiographic findings and prognostic
indicators in patients resuscitated from sudden cardiac death. Circulation 1976;52:895-900. indicators in patients resuscitated from sudden cardiac death. Circulation 1976;52:895-900.
Ruskin JN, DiMarco JP, Garan H. Out-of-hospital cardiac arrest: electrophysiologic observations and selection of long-term antiarrhythmic therapy. N Engl f Med 1980;303:607-13.

DeW'ood MA, Spores J, Notske R, et al. Prevalence of total coronary occlusion during the early hours of transmural myocardial infarction. $N$ Engl f Med 1980;303:877-902.

8 Roberts WC, Jones AA. Quantitation of coronary arterial narrowing at necropsy in sudden coronary death: analysis of 31 patients and comparison with 25 control subjects. Am $\mathcal{F}$ Cardiol 1979;44:39-45

Spain DM, Bradess VA. Sudden death from coronary heart disease: survival time, frequency of thrombi and cigarette smoking. Chest 1970;58:107-10.

10 Davies MJ, Thomas A. Thrombosis and acute coronary-artery lesions in sudden cardiac ischemic death. $N$ Engl $f$ Med 1984;310:1137-40.

11 Friedman M, Manwaring JH, Rosenman RH, Donlon G, Ortega P, Grube SM. Instantaneous and sudden deaths: clinical and pathological differentiation in coronary artery disease. FAMA 1973;225:1319-28.

12 Janse MJ, Kleber AG. Electrophysiological changes and ventricular arrhythmias in the early phase of regional myocardial ischemia. Circ Res 1981;49:1069-81.

13 Mirowski M, Reid PR, Mower MM, et al. Termination of malignant ventricular arrhythmias with an implantable automatic defibrillator in human beings. N Engl f Med 1980;303:322-4. 14 Chamberlain DA, Jewitt DE, Julian DG, et al. Oral mexiletine in high risk patients after myocardial infarction. Lancet 1980;ii:1324-7.

15 Hondeghem LM, Grant AL, Jensen RA. Antiarrhythmic drug action: selective depression of hypoxic cardiac cells. Am Heart $\mathcal{F}$ 1974;87:602-5.

16 Ruskin JN, McGovern B, Garan H, Di Marco JP, Kelly E. Antiarrhythmic drugs: a possible cause of out-of-hospital cardiac arrest. N Engl f Med 1983;309:1302-5

17 Hansteen V, Moinichen E, Lorensten E, et al. One year's treatment with propranolol after myocardial infarction: preliminary report of Norwegian multicentre trial. Br Med $\mathcal{F}$ 1982;284 155-60.

18 Norwegian Multicentre Study Group. Timolol-induced reduction in mortality and reinfarction in patients surviving acute myocardial infarction. $N$ Engl f Med 1981;304:801-7.

19 Beta-Blocker Heart Attack Trial Research Group. A randomized trial of propranolol in patients with acute myocardial infarction. JAMA 1982;247:1707-14.

with acute myocardial infarction. $\mathcal{F} A M A 1982 ; 247: 1707-14$.
20 Lipid Research Clinics. Coronary primary prevention trial results. 1. Reduction in incidence of Lipid Research Clinics. Coronary primary preventio
coronary heart disease. FAMA 1984;251:351-64.

21 Sixty Plus Reinfarction Study Research Group. A double blind trial to assess long term oral anticoagulant therapy in elderly patients after myocardial infarction. Lancet 1980;ii:989-93.

22 Anonymous. Aspirin after myocardial infarction [Editorial]. Lancet 1980;i:1172-3.

\section{Solvent misuse}

The deliberate inhalation of volatile hydrocarbons by children and teenagers is a comparatively recent phenomenon, one of the first reports being by Merry and Zaccariadis in 1962. ${ }^{1}$ The prevalence of solvent abuse in Britain is uncertain and is not restricted to any one area. ${ }^{2}$ It occurs mainly in boys with a peak in those aged 13-15, and is usually a group activity..$^{3-5}$ Boys may take up glue sniffing as a relief from boredom, in response to peer group pressure, out of curiosity, or in an attempt to gain status. Most such youngsters sniff solvents for a short time and on few occasions; only a few become dependent chronic users, and many of these mature out of the solvent habit. ${ }^{356}$ Solvent misuse may, however, also occur in adults. ${ }^{7}$ Press and Done found no relation between solvent abuse and socioeconomic factors ${ }^{8}$ but a higher incidence in children from single parent families and families with paternal unemployment has been found in some studies, ${ }^{35}$ though not in others. ${ }^{9}$

The products that may be used include adhesives, dry cleaning substances, petrol, lighter refills, acrylic paints, fire extinguishing agents, fingernail polish, antifreeze, paint thinners, cleaning fluids, and amyl nitrites. ${ }^{210}$ Adhesives containing toluene and acetone are the most commonly abused. ${ }^{45}$ All these products are readily available, inexpensive, easy to steal, legal, and act quickly; and their effects do not last long. There is no hangover-or the hangover is not as bad as that of alcohol. ${ }^{+}$Glue is most often sniffed from a potato crisp bag; solvents may be directly inhaled from their containers or from a saturated rag. ${ }^{4}$

Volatile solvents are central nervous system depressants. They act quickly because they are absorbed rapidly across the large surface area of the lungs and because of their solubility in lipid. ${ }^{11}$ Subjective effects vary from person to person and depend on the amount of solvent inhaled and the duration of inhalation. The clinical features of solvent intoxication are similar to those of alcohol intoxication with initial stimulation followed by depression. Other symptoms may include euphoria, blurring of vision, tinnitus, slurring of speech, ataxia, feelings of omnipotence, headache, abdominal pain, anorexia, nausea, vomiting, jaundice, chest pain, bronchospasm, impaired judgment, irritability, and excitement. Less often a delirious state is seen with clouding of consciousness, illusions, and hallucinations. ${ }^{2+5}$ Many chronic users report transient symptoms of toxic psychosis, which often have an affective component.12 Convulsions, status epilepticus, ${ }^{13}$ and coma ${ }^{2}$ may occur. Self destructive and antisocial acts may be carried out by people under the influence of solvents. ${ }^{812}$ Psychological dependence and tolerance ${ }^{4}$ may develop, but physical dependence is rare. ${ }^{14}$ Solvent abuse is often associated with abuse of alcohol and multiple illicit drugs. ${ }^{12}$ The effect can be potentiated by prior consumption of alcohol or cigarettes. ${ }^{12}$

Erythematous spots around nose and mouth ("glue sniffer's rash") may be observed when a plastic bag is used for inhalation. ${ }^{5}$ The physical sequels of prolonged solvent abuse have been reported to include aplastic anaemia ${ }^{15}$ and acute hepatic and renal damage. ${ }^{16}$ Irreversible neurological sequelae such as optic atrophy, ${ }^{17}$ encephalopathy, ${ }^{18}$ cerebellar degeneration, ${ }^{19}$ and equilibrium disorders ${ }^{20}$ have been reported in adults who are chronic abusers. Toluene inhalation may cause encephalopathy in children and may lead to permanent neurological damage. "Peripheral neuropathy has been attributed to $n$-hexane inhalation. ${ }^{21}$ The electroencephalogram may show diffuse or focal slow or sharp wave complexes. ${ }^{118}$ Waldron has reviewed the effects of organic solvents as seen in industrial use..$^{22}$

In Britain from 1971 to 1981 some 140 deaths were identified from abuse of volatile substances. ${ }^{23}$ Death rates $N$ were highest in conurbations and in Scotland, Northern Ireland, and northern England. The chief substances were butane $(28 \%)$, solvents in adhesives $(23 \%)$, other solvents $(26 \%)$, aerosols $(15 \%)$, and fire extinguishing agents $(5 \%)$. Most deaths occurred alone at home. In $41 \%$ of cases death was only indirectly associated with solvent abuse (trauma $8 \%$, plastic bag over the head $19 \%$, and inhalation of stomach contents $14 \%$ ). In nearly half death was attributed to the direct toxic effects of the substance; this proportion was highest with aerosols and lowest with solvents in adhesives. ${ }^{23}$ An epidemic of 110 sudden deaths without plastic bag suffocation in American youths was reported by Bass. ${ }^{24}$ Severe cardiac arrhythmias, intensified by hypercapnia, stress, or activity, are thought to be the most likely explanation for sudden death after inhalation of aerosols. ${ }^{24}$ The lack of morbidity and mortality in a group of 300 "glue sniffers" seen at a Glasgow clinic is reassuring. 